\title{
RESPONDING TO CUSTOMERS" “GRAY” REQUESTS: THE SERVICE EMPLOYEE DILEMMA
}

Mary P. Harrison, University of Alabama, USA

William M. Northington, University of Alabama, USA

Sharon E. Beatty, University of Alabama, USA

Betsy B. Holloway, Samford University, USA

Sijun Wang, Loyola Marymount University, USA

\begin{abstract}
This research examines how service employees make the decision to comply with customer requests. In this paper, a unique type of customer request is studied: the "gray" request, a request in which the employee is asked to do something that is outside of their job description or outside of what they would normally do to deliver the service (but not something that is fraudulent or illegal). This research develops the gray request concept and provides a conceptual model to explain the circumstances by which gray requests occur and employees comply. Twenty-three qualitative interviews with employees from two different service firms in different industries are conducted. Three main motivational categories relating to why employees respond favorably to gray customer requests emerge: (1) the desire to help others (2) the desire to help the company and (3) the desire to help themselves. The organizational, customer, employee, and situational dimensions that determine when and if employees will respond favorably include: (1) the organization's culture, the norm of flexibility, and the reward/incentive system (2) the customer demographics (i.e., loyal customer; elderly) (3) the personality factors of the employee (i.e., allocentrism) and (4) the situational factors (ability, ease, legality, safety, time constraints, type of request, and urgency).
\end{abstract}

